\title{
DESIGN AND OPERATION OF THE CRYOSTAT FOR THE CESR-C SUPERCONDUCTING WIGGLER MAGNETS
}

\author{
Y. He ${ }^{\#}$, G. Codner, R.D.Ehrlich, Y. Li, V. Medjidzade, A. Mikhailichenko, \\ N. B. Mistry, E. Nordberg, D. Rice, D. Sabol, K. Smolenski, D. Widger \\ Laboratory for Elementary-Particle Physics, Cornell University, Ithaca, NY 14853, USA \\ E. N. Smith, Laboratory of Atomic and Solid State Physics, Cornell University
}

\begin{abstract}
$^{*}$
As part of the "CESR-c" upgrade project, a full-scale prototype superferric wiggler module has been installed into the Cornell Electron Storage Ring (CESR) and successfully operated since October 2002. At least eleven more wigglers are to be installed to enable full operation of the CESR-c. The wiggler magnets are chosen to achieve the necessary damping for optimum operation of CESR-c at a beam energy of $1.88 \mathrm{GeV}$. The wiggler cryostat units are designed to be modular, of fixed length flange-to-flange, so that they can be easily installed and exchanged. The cryostat incorporates several novel features including a suspension system with very low heat leak that prevents any significant motion during cool down, a custom triple cryogenic feedthrough, and a helium vessel made in two halves, each enclosing a halfmagnet. This article presents the design, fabrication, and operation of the CESR-c wiggler cryostat.
\end{abstract}

\section{INTRODUCTION}

The conversion program CESR-c will extend CESR operating range to $1.5 \mathrm{GeV}$ through $5.6 \mathrm{GeV}$ [1]. It requires the addition of over 15 meters of wiggler magnets to enhance synchrotron radiation (SR) effects for optimum CESR operation at low energies. The wiggler design is of superferric type [2][3]. The wiggler cryostat units are designed to be modular of fixed length flange-toflange, so that they can be easily installed or replaced. A full-scale prototype wiggler has been installed into the CESR ring and successfully operated since October 2002. Five more wigglers have been manufactured and tested and are now installed. At least six more wigglers are to be installed to enable full operation of the CESR-c [4].

The cryostat serves to support the cold mass accurately and reliably within the vacuum vessel, to provide all required cryogenic plumbing, and to insulate the cold mass from heat radiated and conducted from the environment. The fact that the cryostat should fit into the tight space available in the existing CESR tunnel while providing maximum wiggler magnetic length makes its design more challenging.

The cryostat is $0.6 \mathrm{~m}$ in diameter and $1.725 \mathrm{~m}$ in length. It consists of a liquid helium vessel made in two halves, insulation vacuum vessel, thermal shields,

\footnotetext{
* work supported by the US National Science Foundation \#yh63@cornell.edu
}

helium stack for housing current leads and instrumentation wires, suspension system, cryogenic plumbing and an integral copper beam-pipe with warmbore (see Fig. 1, 2).

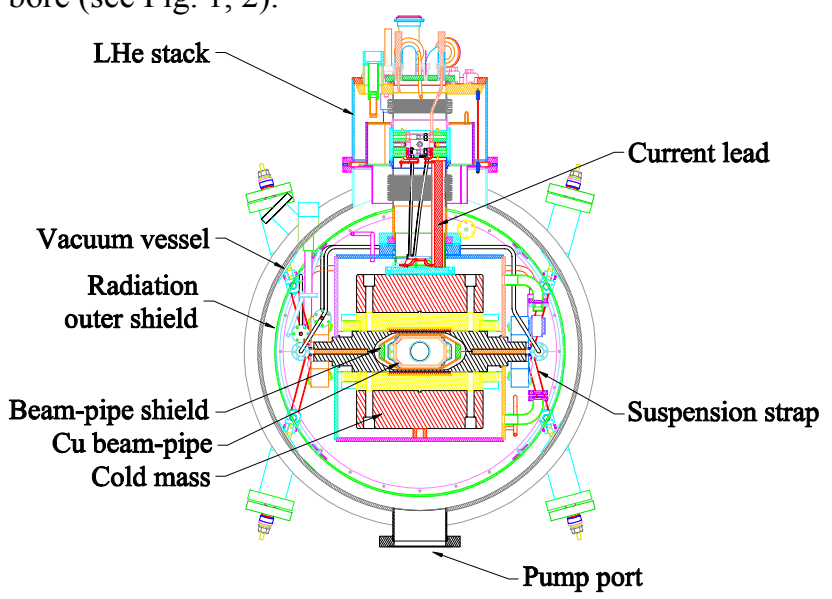

Figure 1: Wiggler cryostat cross-section.

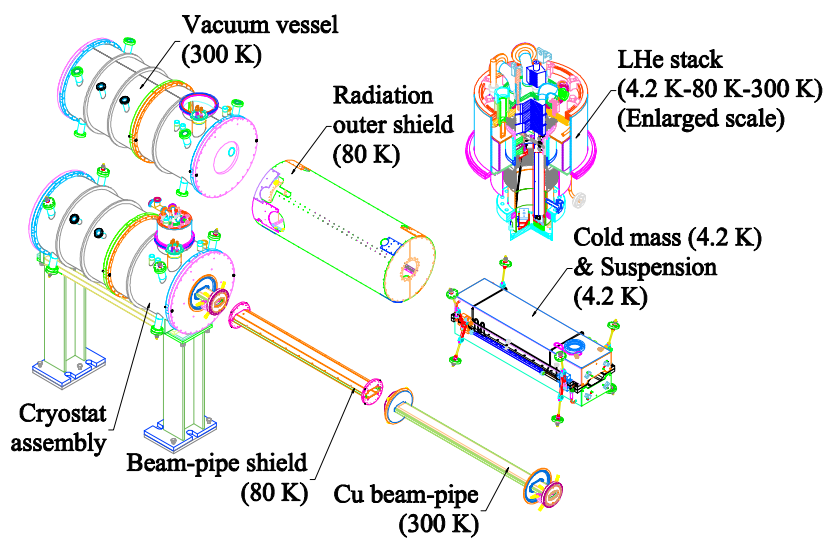

Figure 2: Cryostat assembly with major components.

\section{DESIGN DESCRIPTION}

\section{Thermal Design Considerations}

In addition to mechanical requirements, the cryostat is designed to minimize heat transfer by radiation and conduction from ambient to the cold mass. The heat transfer to the cold mass directly affects the magnet's operating cost.

The conductive heat leak is minimized by choosing the proper materials for the cold mass support, employing thermal interception at $80 \mathrm{~K}$ and providing high vacuum insulation. The radiative heat transfer is reduced by 
interposing $\mathrm{LN}_{2}$ cooled thermal shields and wrapping the cold mass with multilayer super-insulation. The thermal load associated with the current leads is reduced by the use of high temperature superconducting (HTS) current leads below $77 \mathrm{~K}$. The estimated static heat leaks to the stages at $4.2 \mathrm{~K}$ and $80 \mathrm{~K}$ are summarized in table 1.

Table 1: Static heat leak in cryostat (in Watts)

\begin{tabular}{|l|l|l|}
\hline Components & $\mathbf{4 . 2} \mathbf{~ K}$ & $\mathbf{8 0 ~ K}$ \\
\hline Thermal shields & 0.35 & 6.8 \\
\hline Supports & 0.05 & 9.0 \\
\hline Cryogenic feedthrough & 0.14 & 3.2 \\
\hline Current leads & 0.17 & 17.1 \\
\hline LHe stack & 0.19 & 1.4 \\
\hline Total & $\mathbf{0 . 9}$ & $\mathbf{3 7 . 5}$ \\
\hline
\end{tabular}

\section{Cold Mass and Suspension System}

The magnet has $1.3 \mathrm{~m}$ active length with a peak field of $2.1 \mathrm{~T}$. It is made in two halves. Each half-magnet has a mass of about $420 \mathrm{~kg}$ cooled in a helium bath. The two halves are connected through interconnecting side tubes for wires and LHe flow.

The cold mass is suspended in a $609 \mathrm{~mm}$ diameter vacuum vessel by eight tension straps with four straps at each of the two ends (see Fig. 3). With this suspension scheme the center of cold mass does not move during cool down. The racetrack-shaped straps are made of S-glass fiber in an epoxy matrix, which has a low conductivity-toyield-strength ratio. The warm end of the strap is joined to an invar rod through a clevis, which is thermally anchored to the $80 \mathrm{~K}$ thermal shield by copper braids. The alignment of the cold mass axis relative to the beam-pipe is performed at room temperature by adjusting nuts on the invar rods from the outside of the vacuum vessel.

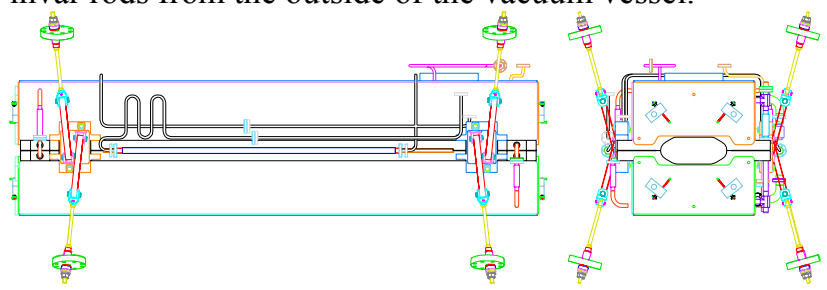

Figure 3: Cold mass suspension system.

\section{Radiation Shields, beam-pipe and interconnection}

Radiation shields are interposed to intercept radiative heat transfer between cryostat vessel and cold mass, and between beam-pipe and cold mass. The shields are made of pure aluminum $1100-\mathrm{O}$ with a thermal conductivity of $300 \mathrm{Wm}^{-1}-\mathrm{K}^{-1}$ at $80 \mathrm{~K}$. The outer radiation shield is cooled by liquid nitrogen through stainless steel (SS) tubes which are clamped and glued to provide good thermal contact. The beam-pipe shield is cooled through thermal conduction from the outer shield via end flanges.
The ultrahigh vacuum beam-pipe is made of copper with water cooling channels at both sides to intercept wiggler radiation.

The cold mass, radiation shields and beam-pipe are interconnected at both ends through thermally insulating supports which allow adjustment during assembly and accommodate shrinkage during cool down. At each end, the radiation shield is supported from the cold mass by four tensioned Kevlar straps while the beam-pipe is supported by four fiberglass rods from the shield.

The cold mass, radiation shields and beam-pipe are all wrapped with four to ten layers of multilayer superinsulation made of aluminized Mylar with mesh polyester interlayer.

\section{LHe Stack and Instrumentation}

A LHe stack on the top of vacuum vessel houses HTS current leads, instrumentation wires, voltage taps and trim current leads. A liquid nitrogen pot provides an $80 \mathrm{~K}$ heat sink for thermal intercept.

A pair of HTS leads is used to deliver the current to the magnet coils. The warm end of the lead is thermally anchored at the $\mathrm{LN}_{2}$ pot and the cold end at $4 \mathrm{~K}$.

When a magnet quenches, part of the generated heat is transferred into the helium bath and thus the helium pressure is increased. The stack is equipped with a safety relief valve and burst disc to ensure fast discharge of helium due to quench or cryogenic failure.

Thick film $56 \mathrm{k} \Omega$ resisters are placed at various locations to monitor the temperature. They are mounted on copper tabs, with leads wrapped several turns around the tab to ensure a good thermal contact.

Two LHe level sensors are used, with one in the stack and the other installed in the side tube of the cold mass. A $\mathrm{LN}_{2}$ level sensor is installed in the stack $\mathrm{LN}_{2}$ pot at the output of the radiation shield cooling line.

\section{Cryogenic Plumbing}

A triple cryogenic feedthrough with $\mathrm{LN}_{2}$ intercepted vacuum break accommodates LHe input, Helium cold gas return and $\mathrm{LN}_{2}$ input. The low heat leak compact design allows for a flanged bellows connection in the tight space in the CESR tunnel.

The cryogenic plumbing contains four separate circuits: LHe line; gaseous helium warm return; $\mathrm{LN}_{2}$ line for shields and $\mathrm{LN}_{2}$ pre-cooling line for cold mass. They are all made of SS tubing.

The $\mathrm{LN}_{2}$ precooling circuit is clamped and glued onto $9.525 \mathrm{~mm}$ thick copper plates which are thermally bonded between SS support plates of cold mass halves. This line cools the cold mass to $80 \mathrm{~K}$ from room temperature and is capped off afterwards. The gaseous helium warm return line serves for cooling down from $80 \mathrm{~K}$ to $4.2 \mathrm{~K}$. It is also capped off afterwards. The helium output is then switched to the cold return line which is connected to the refrigerator through the transfer line. The $\mathrm{LN}_{2}$ precooling line and helium warm return line are anchored at $80 \mathrm{~K}$ to minimize $4.2 \mathrm{~K}$ heat load during wiggler operation. 


\section{Vacuum System}

There are two separate vacuum systems in the wiggler module. The cryogenic insulation vacuum provides a high vacuum to avoid heat load by conduction from the environment to the cold mass, while the beam pipe vacuum requires ultrahigh vacuum to provide a good beam lifetime and low background for the experiment.

The insulation vacuum of the prototype wiggler was pumped by a turbomolecular pump. All future wigglers are pumped by diffusion pumps with water cooled baffles. Once the insulation space is pumped to the range of $10^{-4} \mathrm{~Pa}, \mathrm{LN}_{2}$ precooling process can be started. After the system is cooled down, the cryopumping on the large surface of multilayer super-insulation is sufficient for maintaining high vacuum $\left(10^{-6} \mathrm{~Pa}\right)$.

A non-evaporable getter (NEG) pump is connected to the copper beam-pipe to pump gas load induced by wiggler SR and bending magnet SR.

\section{ASSEMBLY AND TEST}

\section{Assembly}

Vacuum leak checking, including cold shocking of cryogenic subassemblies at $77 \mathrm{~K}$, was carried out throughout the cryostat manufacture process.

The insulation vacuum vessel is constructed in two parts to facilitate cryogenic plumbing connection. The short vessel incorporates all necessary ports for cryogenic plumbing and instrumentation wiring. The long vessel is installed last when all components are assembled and aligned. Finally two $660 \mathrm{~mm}$ O-ring sealed flanges are welded onto the beam-pipe to complete the cryostat. The assemble sequence is as below (see Fig. 4):

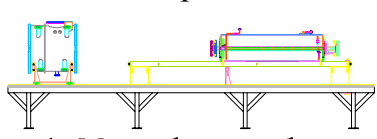

1. Mount short vessel
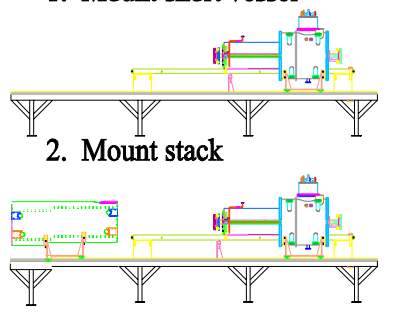

3. Mount radiation shield
2. Mount stack

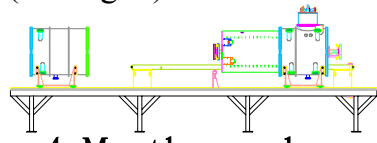

4. Mount long vessel

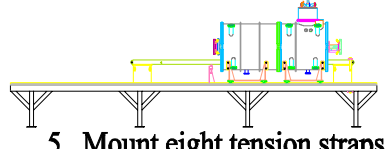

5. Mount eight tension straps

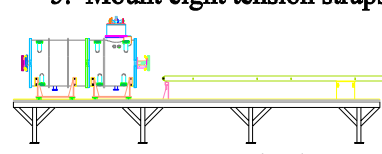

6. Remove strongback
Figure 4: Assembly procedure.

Pre-assembly: Place preassembled unit of beam-pipe shield with beam-pipe on the bottom half of cold mass, and then connect the top half to the bottom half with precooling plates in between;

Step 1 and 2: Slide short vacuum vessel over cold mass and connect cryogenic tubing. Assemble current leads and wires to the cold mass, and then finish stack assembly.

Stage 3, 4 and 5: Install outer radiation shield and connect liquid nitrogen tubing. Slide long vacuum vessel into place, mount all eight tension straps with invar rods.
Stage 6: Remove temporary support from the cold mass. Finish end interconnection, and then weld the vessel end flanges to beam-pipe;

Throughout the assembly optical surveying is used to provide alignment of the magnet to the beam-pipe within $0.1 \mathrm{~mm}$.

\section{Test and Operation}

Prior to installation in the CESR tunnel, each wiggler magnet was tested and run above $2.1 \mathrm{~T}$ field. Magnetic field quality measurements were performed.

Three proportional pneumatic control valves regulate LHe input, cold helium gas return, and $\mathrm{LN}_{2}$ input. The input flow of $\mathrm{LHe}$ and $\mathrm{LN}_{2}$ are controlled through level sensors. Helium bath pressure is controlled using helium return valve and a pressure sensor. LHe overpressure or power outage would shut the LHe input valve and the cold return valve.

For protecting the magnet, the power supply current would commence a slow run-down if one of three cryogenically related indications occurs: high He pressure in cold mass; high $\mathrm{He}$ pressure in return line; poor insulation vacuum in cryostat. More importantly, quenches cause external switch to open ramping magnet down in $\sim 10$ seconds.

So far we have completed and tested six wigglers. The prototype was installed into the CESR ring and successfully operated with beam since October 2002. All six wigglers worked well the first time they were cooled down. One to three training quenches occurred to some of them before reaching the target current. It took 48 hours to precool the cold mass to $80 \mathrm{~K}$ using the $\mathrm{LN}_{2}$ precooling line. Then it took five hours and 200 liters of LHe to cool down to $4.2 \mathrm{~K}$ and fill up the cold mass. The static LHe evaporation rate is about $1.5 \sim 2.0 \mathrm{l} / \mathrm{h}$. This corresponds to a heat load of $1.0 \sim 1.35 \mathrm{~W}$, close to the budgeted load. When quenches did occur, the maximum pressure in helium bath went up to 1.8 atmospheres in a few seconds with a helium loss of 16 liters.

\section{SUMMARY AND ACKNOWLEDGMENTS}

A full-scale superferric wiggler prototype, to enhance synchrotron radiation damping for CESR-c at low energies, has been successfully developed, fabricated and operated in the CESR ring. Five more wigglers have been tested and are now installed. Proper design of the cryostat plays an important role in ensuring reliable wiggler magnet operation.

The authors would like to thank the machine shop and technical support staff at LEPP for their contributions to the manufacture of the wiggler cryostats.

\section{REFERENCES}

[1] D. Rice, Proc. EPAC 2002, p. 428

[2] A. Mikhailichenko, Proc. PAC 2001, p. 3648

[3] J. Crittenden et al, PAC 2003, WPAE009

[4] D. Rice et al., PAC 2003, TOAB007 